\title{
Double Acl Reconstruction Failure in A Young Soccer Player In Treatment with Retinoids: A Case Report
}

\author{
Marcheggiani Muccioli Giulio Maria*1, Lullini Giada ${ }^{1}$, Rinaldi Vito Gaetano ${ }^{1}$, Tsapralis Kyriakos ${ }^{2}$ and \\ Zaffagnini Stefano ${ }^{1}$ \\ ${ }^{1}$ IRCCS Istituto Ortopedico Rizzoli, University of Bologna, Italy \\ ${ }^{2}$ Isokinetic FIFA Medical Center of Excellence, Italy
}

*Corresponding author: Marcheggiani Muccioli Giulio Maria, IRCCS Istituto Ortopedico Rizzoli, University of Bologna, Italy

\begin{tabular}{|c|c|}
\hline ARTICLE INFO & ABSTRACT \\
\hline Received: 蔧 March 11, 2019 & Citation: Marcheggiani Muccioli Giulio M, Lullini G, Rinaldi Vito G, Tsapralis K, Zaffagnini \\
\hline Published: March 25, 2019 & $\begin{array}{l}\text { S. Double Acl Reconstruction Failure in A Young Soccer Player In Treatment with Reti- } \\
\text { noids: A Case Report. Biomed J Sci \& Tech Res 16(2)-2019. BJSTR. MS.ID.002836. }\end{array}$ \\
\hline
\end{tabular}

\section{Introduction}

Isotretinoin is a vitamin A derivative commonly used for the treatment of severe acne. Although being effective, long-term or excessive use of isotretinoin has some toxic or side effects on the musculoskeletal system. The most frequent effects are arthralgia, myalgia with hyperostosis, reduced bone mineralization and calcifications in ligaments and tendons, tendinopathies and periosteal proliferations [1]. Excess of vitamin A intake has been shown to result in bone reabsorption via increasing the number and size of osteoclasts and decreasing osteoid surface. Retinoid- induced side effects on the musculoskeletal system can occur during both shortand long-term administration [2]. To date literature on retinoids effects on tendons is scarce [3] and to the best of authors' knowledge studies on soft tissues integration in patients treated with vitamin A derivates were never conducted. We present the case of a double anterior cruciate ligament (ACL) reconstruction failure in a young amateur soccer player patient in treatment for severe cystic acne with isotretinoin.

\section{Case Report}

A 16 years old amateur soccer player first came to our attention in 2016. The patient experienced right knee ACL lesion following a non-contact knee sprain occurred during a soccer game. Patient's medical history revealed no other musculoskeletal diseases or comorbidity and investigations for collagen pathologies were negative. Magnetic Resonance Imaging (MRI) showed an isolated complete ACL lesion. One month later the patient underwent an ACL reconstruction with lateral plasty using autologous hamstrings. Surgery was performed arthroscopically by an expert surgeon with an operating volume of more than 50 ACL reconstructions yearly. Over-the-top with later plasty technique was used to reconstruct ACL [4]. Postoperatively, the patient started rehabilitation the day after surgery. Rehabilitation was performed in a certified rehabilitation center (FIFA medical center of excellence) with an experienced physiotherapist, following a standard rehabilitation protocol. Progressive weight bearing was allowed, [5] and full range of motion was achieved in 45 days. The post-operative recovery was good but four months after surgical intervention the patient underwent a second knee sprain during the on-field rehabilitation with no evident trauma. MRI showed a lack in the graft integration inside the tibial tunnel (Figures 1-3). Clinically Lachman and anterior drawer were positive, and no meniscal tears were detected. The ACL revision surgery was then scheduled and performed by the same expert surgeon by means of Achilles tendon allograft with Over-the-top technique with lateral plasty $[4,5]$. 

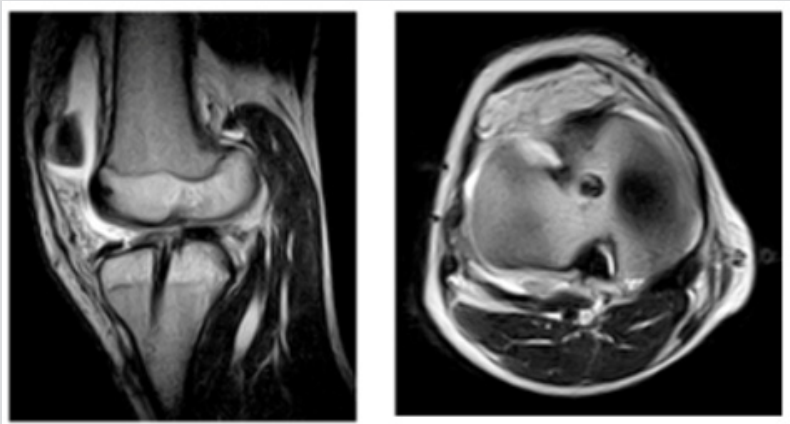

Figure 1: First ACL Reconstruction Failure 4 months after the surgery.
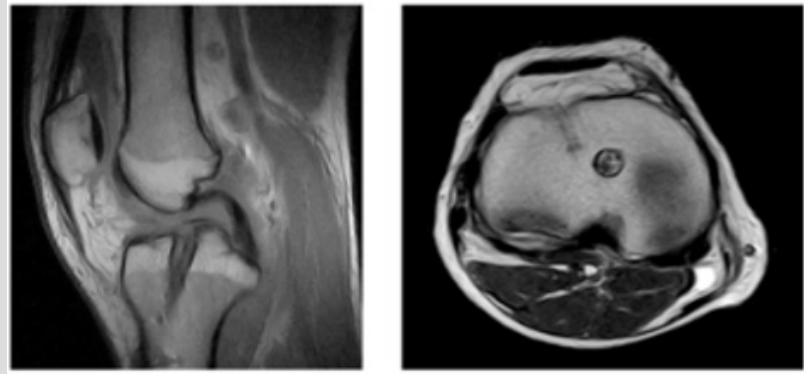

Figure 2: ACL Revision Failure 8 months after the surgery.
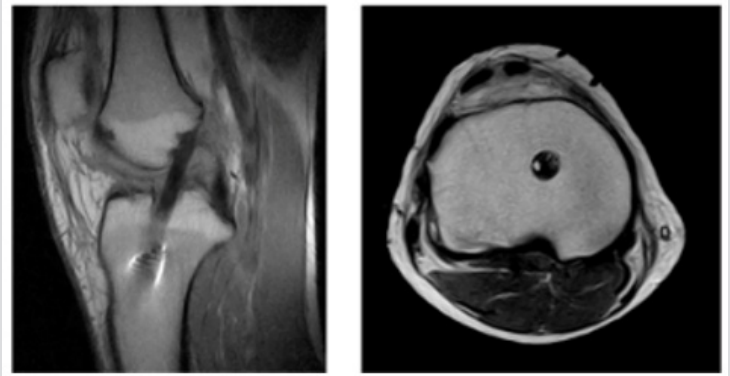

Figure 3: Anatomic ACL Reconstruction with autologous bone-patellar tendon-bone

(BPTB) 12 months after the surgery.

Two days after surgery the patient began rehabilitation. Progressive weight bearing was granted. During rehabilitation quadricipital strengthening exercises were performed and full Range of Motion (ROM) was achieved in two months. The patient did not practice sports for eight months after surgical intervention. Eight months after the revision surgery the patient experienced a second ACL reconstruction failure with no evident trauma occurring. MRI showed again no graft-tissue integration inside bone tunnel. Patient medical history was then deeply investigated revealing a 2-years lasting therapy with retinoids for acne. This therapy was then suspended, and a second revision surgery was scheduled. Anatomic ACL reconstruction with autologous bonepatellar tendon-bone (BPTB) was performed by the same surgeon. At 9 months follow-up the patient successfully completed the rehabilitation program and fully recovered sports activity. MRI performed 12 months after the second revision surgery showed BPTB graft integration into bone tunnels. At 18 months follow-up the patient was routinely playing soccer and no ACL reconstruction failure was reported.

\section{Discussion}

The first surgery was performed by an expert surgeon in ACL reconstruction using "Over-the-top Plus Extra-Articular Lateral Tenodesis With Hamstring Tendon Grafts" technique. This surgical technique, with more than 20 years follow-up, is daily performed at our hospital. The graft used was the semitendinosus and gracilis tendons, sutured as a double-stranded graft. The tibial insertion was left intact. The tendons were passed through the tibial tunnel and "over the top" of the femur. Ten cycles of knee flexion-extension were performed for graft pretensioning. A groove was made with an osteotome on the lateral aspect behind the lateral epicondyle of the femur to freshen the bone in order to enhance graft healing. The knee was flexed to $90^{\circ}$ with $45^{\circ}$ of external tibial rotation, and a posterior drawer was applied. The tendons were then fixed to the cortical bone of the femur with 2 bone staples under manual maximum tension. The remaining part of the graft was then passed deep to the iliotibial band, superficial to the lateral collateral ligament, and fixed with a single staple onto Gerdy's tubercle as extra-articular plasty with the knee flexed at $70^{\circ}$ [4].

In a recent study, Zaffagnini et al. [4], demonstrated that this ACL reconstruction technique has a good outcome in terms of laxity control, with 4 out of 29 clinical failures and 1 re-rupture among 52 patients at 20-year minimum follow-up and very good success rates, with $84.3 \%$ normal or nearly normal knees measured with the IKDC rating system at this follow-up. The ACL revision intervention was performed by the same surgeon by means of Achilles tendon allograft with the same consolidated technique employed for the first reconstruction. Considering the correct surgical performance of ACL reconstruction, the surgeon expertise, the proven reliability of the used technique even in the long term, the reliability of applied rehabilitation protocol and the lack of an important trauma causing the two revision failures, this case catched authors attention. Therefore, we decided to perform a deeper clinical investigation with an accurate patient medical history analysis, and we found out that the patient had been in treatment with isotretinoin for about 18 months before surgery.

Isotretinoin is a vitamin A derivative commonly used for the treatment of severe acne. Although being effective, the use of isotretinoin can cause toxic or side effects on different organs included the musculoskeletal system during both short- and long-term administration [1]. High levels of vitamin A can cause arthralgia, myalgia with hyperostosis, diminished bone mineralization and calcifications in ligaments and tendons, tendinopathies and periostal proliferations [2]. Retinoic acid can be involved in lack of graft osteointegration [6]. As early as 3-7 
days after the tendon is placed, the gap between the tendon graft and bone tunnel and the tendon-bone interface is infiltrated with loose and poorly organized fibrovascular tissue. An inflammation reaction characterized by an influx of cells, such as fibroblasts, vascular endothelia, neutrophils, and macrophages, is obvious within the tendon-bone interface with the expression of various growth factors and cytokines, such as type III collagen, vascular endothelial growth factor (VEGF), fibroblast growth factor (FGF), and bone morphogenetic proteins (BMPs).At around 1-2 weeks, the interface tissue increases in cellularity and vascularity, with an increased number of osteoblasts, chondroid cells and fibroblasts around the bone wall. Bone-growth factors, such as BMPs, are aggressively expressed and newly formed trabecular bone can be seen sparsely near the bone wall. Approximately 4 weeks after the operation, the fibro-vascular interface tissue becomes denser and better organized and is gradually degraded by the chondroid cells from the bone into the tendon graft with a deposition of type II collagen into the matrix, resulting in newly formed lamellar bone and early fibrocartilage progressively growing into the interface tissue. The bone wall surrounding the tendon also becomes thicker as a result of the new bone formation [7]. However, during this stage, the collagen fibers are still poorly organized, and the continuity between the tendon and bone can just occasionally be seen at this period. Later, at 6 weeks, the collagen fibers become more mature, and the continuity between the tendon and bone is re-establiushed. Around 8 weeks after the operation, perpendicular oriented fibers resembling Sharpey's fibers can be seen along the axis of the tendon in the interface. These Sharpey-like fibers are composed of type III collagen and connect the bone and the tendon graft and are regarded as the earliest sign of osteointegration into the tendon. As a result, the biomechanical outcomes of tendon healing at the early stage are essentially dependent on collagen fiber continuity and tendon osteointegration [8]. Literature has shown that retinoic acid can act on collagen production, hindering and delaying it. In the reported case lack of graft osteointegration can be due to retinoic acid systemic treatment. Nelson DL et al. [9] indicated that retinoic acid has an effect on the growth of human dermal fibroblasts and on the elaboration and processing of proteins of the extra-cellular connective tissue matrix.

Jetten et al. [10] showed that retinoids inhibit proliferation, reduce saturation density, and alter cell morphology of fibroblastic cells. Since collagen is the major protein secreted by fibroblasts, we supposed that retinoic acid can interfere with both tendon healing and osteointegration at the insertion level and at the bone tunnel interface, hindering the production of collagen. Clinical studies showed that retinoic acid acts on the dermal Retinoic acid receptors (RAR), accelerating the keratin turnover process, eliminating the epidermal horny layer and therefore causing skin dryness and fragility. If the same process occurs to the tendon this event can produce a greater fragility. Webb et al. [11] demonstrated in a in-vitro study the presence of RAR receptors in tendons and found out that Vit. A derivates, including retinoic acid, determine downregulation of the tendon stem cells differentiation, leaving the tendon to an immature cellular stage. This important finding can support the hypothesis that retinoic acid can negatively interact on the ligamentization process consisting in an initial avascular necrosis of the graft during the first 3 weeks because of the adverse intra-articular environment, followed by a new ligament cells repopulation phase, thanks to stem cells differentiation.

\section{Conclusion}

Retinoic acid effects on cartilage and bones have previously been shown in the literature. The reported case of a young soccer player in therapy with retinoids undergoing ACL reconstruction shows a lack in soft-tissue graft integration. Medication suspension seems to lead to graft integration success according to MRI at 12 months follow-up and to clinic at 18 months follow-up. Effects of retinoic acid on soft tissues and tendons need to be further investigated.

\section{References}

1. DiGiovanna JJ (2001) Isotretinoin effects on bone. J Am Acad Dermatol 45(5): S176-182.

2. De Oliveira HTR, Bergoli RD, Hirsch WDB, Chagas OL, Heitz C, et al. (2013) Isotretinoin effect on the repair of bone defects - a study in rat calvaria. J Craniomaxillofac Surg 41(7): 581-585.

3. Ylldızgören MT, Karataş Toğral A, Baki AE, Ekiz T, et al. (2015) Effects of isotretinoin treatment on cartilage and tendon thicknesses: an ultrasonographic study. Clin Rheumatol 34(7): 1255-1258.

4. Zaffagnini S, Marcheggiani Muccioli GM, Grassi A, Roberti di Sarsina T, Raggi F, et al. (2017) Over-the-top ACL Reconstruction Plus ExtraArticular Lateral Tenodesis With Hamstring Tendon Grafts: Prospective Evaluation With 20-Year Minimum Follow-up. Am J Sports Med 45(14): 3233-3242.

5. Zaffagnini S, Grassi A, Marcheggiani Muccioli GM, Roberti Di Sarsina T, Macchiarola L, et al. (2018) Anterior cruciate ligament revision with Achilles tendon allograft in young athletes. Orthop Traumatol Surg Res 104(2): 209-215.

6. Hotchkiss CE, Latendresse J, Ferguson SA (2006) Oral treatment with retinoic acid decreases bone mass in rats. Comp Med 56: 502-511.

7. Panni AS, Milano G, Lucania L, Fabbriciani C (1997) Graft healing after anterior cruciate ligament reconstruction in rabbits. Clin Orthop Relat Res, pp. 203-212.

8. Tekin NS, Ozdolap S, Sarikaya S, Keskin SI (2008) Bone mineral density and bone turnover markers in patients receiving a single course of isotretinoin for nodulocystic acne. Int J Dermatol 47(6): 622-625.

9. Nelson DL, Balian G (1984) The effect of retinoic acid on collagen synthesis by human dermal fibroblasts. Coll Relat Res 4(2):119-28.

10. Jetten AM, Jetten ME, Shapiro SS, Poon JP (1979) Characterization of the action of retinoids on mouse fibroblast cell lines. Exp Cell Res 119(2): 299.

11. Webb S, Gabrelow C, Pierce J, Gibb E, Elliott J, et al. (2016) Retinoic acid receptor signaling preserves tendon stem cell characteristics and prevents spontaneous differentiation in vitrox. Stem Cell Res Ther 7: 45. 


\section{ISSN: 2574-1241}

DOI: $10.26717 /$ BJSTR.2019.16.002836

Marcheggiani Muccioli Giulio M. Biomed J Sci \& Tech Res

(C) (P) This work is licensed under Creative

Submission Link: https://biomedres.us/submit-manuscript.php

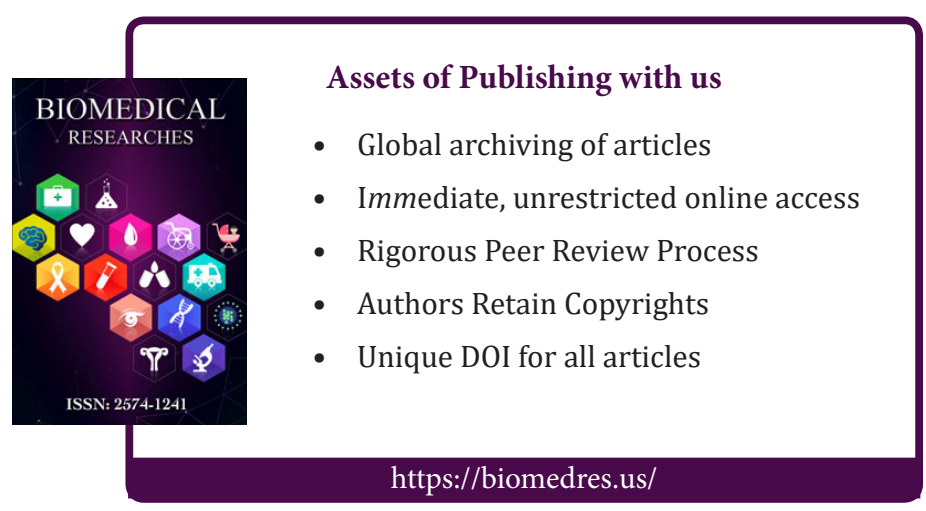

